\title{
Intelligent Routing Techniques for Mobile Ad hoc Networks using Swarm Intelligence
}

\author{
CH. V. Raghavendran \\ Research Scholar, Dept of Computer Science, Adikavi Nannaya University, Rajahmundry, India \\ raghuchv@yahoo.com \\ G. Naga Satish \\ Research Scholar, Dept of Computer Science, Adikavi Nannaya University, Rajahmundry, India \\ gantinagasatish@gmail.com \\ P. Suresh Varma Prof. \\ Professor, Dept of Computer Science, Adikavi Nannaya University, Rajahmundry, India \\ vermaps@yahoo.com
}

\begin{abstract}
A Mobile Ad hoc Network (MANET) is a collection of autonomous self-organized nodes. They use wireless medium for communication, thus two nodes can communicate directly if and only if they are within each other's transmission radius in a multi-hop fashion. Many conventional routing algorithms have been proposed for MANETs. An emerging area that has recently captured much attention in network routing researches is Swarm Intelligence (SI). Besides conventional approaches, many new researches have proposed the adoption of Swarm Intelligence for MANET routing. Swarm Intelligence (SI) refers to complex behaviors that arise from very simple individual behaviors and interactions, which is often observed in nature, especially among social insects such as ants, bees, fishes etc. Although each individual has little intelligence and simply follows basic rules using local in formation obtained fro $m$ the environ ment. Ants routing resembles basic mechanisms from distributed Swarm Intelligence (SI) in biological systems and turns out to become an interesting solution where routing is a problem. Ants based routing is gaining more popularity because of its adaptive and dynamic nature. A number of Swarm Intelligence (SI) based algorithms were proposed by researchers. In this paper, we study bio-inspired routing protocols for MANETs.
\end{abstract}

Keywords - Mobile Ad hoc Network, Swarm Intelligence, Ant Colony Optimization, Bee Hoc Optimization, Routing.

\section{Introduction}

In Mobile Ad hoc Networks (MANET) [14][15], nodes are self-organized and use wireless links for communication between themselves. They dynamically form a temporary network without using any existing network infrastructure or centralized administration. These are often called infrastructure-less networking since the mobile nodes in the network dynamically establish routing paths between themselves. Examples are conference, battlefield, rescue scenarios, sensor networks placed in an area to monitor the environ ment, mesh networks for wireless Internet access etc. Routing solutions must address the nature of the network, and aim at minimizing control traffic, to preserve both bandwidth and energy at nodes.

One of the major issues that affects the performance of an ad hoc network is the way routing is implemented in a network. Routing algorithms used in conventional wired networks is impractical in ad hoc networks due to its inability to adapt to the changing topology in a mobile environment. Generally, routing is the process of discovery, selecting, and maintaining paths from a source node to destination node deliver data packets. The goal of every routing algorithm is to direct traffic from sources to destinations, maximizing network performance whilst minimizing costs. This is a main challenge in MANET. Because the MANET possesses dynamic and random characteristics. Nodes move in an arbitrarily manner and at changing speed, often resulting in connectivity problems. The high mobility and the arbitrarily movement of nodes in MANET causes links between hosts to break frequently.

Routing protocols for MANETs are classified into three categories [16][17]: proactive, reactive and hybrid. Proactive routing protocols exchange control packets among mobile nodes and continuously update their routing tables. This causes high overhead congestion of the network and requires lots of memory. The advantage of proactive protocols is that nodes have correct and updated information. Reactive routing 
protocols establish a route to the destination when it is needed. The advantage of these protocols is that the routing tables located in memory are not continuously updated. The aim of these protocols is to save time in the route discovery process, since the reactive protocol is designed to reduce the latency which is critical in this kind of protocols. Hybrid protocols use a combination of both proactive and reactive activities to gather routes.

Researchers have proposed several routing protocols designed to provide communication in wireless environment, such as AODV [18], DSDV [17], ZRP [19], LAR [20], OLSR and DYMO [21] etc. Nature inspired routing protocols have been becoming the focus of research because they achieve the complex task of routing through simple agents which traverse the network and collect the routing information in an asynchronous fashion. The changing and unpredictable nature of MANETs poses a wide range of challenges for effective routing. This paper attempts to study different routing algorithms for MANETs inspired from the nature that are developed based on Ant Colonies, and Bee Colonies.

The first algorithm which presented a detailed scheme for MANET routing based on ant colony principles is $A R A$ [22]. The algorith $\mathrm{m}$ has its roots in $A B C$ [23] and AntNet [24] routing algorith ms for fixed networks, which are inspired by the pheromone laying behavior of ant colonies. The algorith $\mathrm{m}$ floods ants to the destinations while establishing reverse links to the source nodes of the ants. AntHocNet has been recently proposed in [25] which is a hybrid algorithm having both reactive and proactive components. The algorithm tries to keep most of the features of the original AntNet and shows promising results in the simulation tests over $A O D V$. Termite is another MANET routing algorithm inspired from termite behavior [26]. Recently, Wedde, Farooq and Zhang have proposed a novel routing algorithm for fixed networks which is inspired by forag ing principles of honey bees [27]. The algorithm is simple but delivers the same/better performance as that of AntNet [24].

This paper examines the qualitative comparisons and challenges of routing protocols for MANET based on the technique of Swarm Intelligence. The rest of the paper is organized as follows. Section II introduces the Swarm Intelligence. Section III discusses the Ant Colony based algorithms. Section IV discusses Bee inspired routing techniques. Finally Section V represents the conclusion of this paper.

\section{Swarm Intelligence}

In nature several animals tend to live in large swarms like insect colonies, bird flocks or fish schools. The reason is that in the swarm each animal is more effective for evolution than single animals. Many social insects like ants, bees, termites, or wasps live in colonies or hives. They exhibit an astonishingly welldeveloped social behavior and are able to self-organize, even in the absence of a central leader like a queen. Honey bees communicate locations of food sources by the language of dance that is understood by all nearby honey bees. On the other hand, many insects use a form of indirect communication called stigmergy. Stig mergy works by leaving traces in the environment that can be understood by other insects. Termites use stigmergy to build complex nests by simple rules. A termite constructing a nest deposits material like a mud ball and invests it with pheromones, a chemical that can be smelled by other termites. The smell of pheromones encourages other termites to deposit their material close to freshly deposited pheromones. This way, a group of termites can manage to synchronize so that they all work on the same spot.

Swarm Intelligence (SI) [1] is an Artificial Intelligence technique based on the study of collective behavior in decentralized, self-organized systems. The expression "swarm intelligence" was introduced by Beni \& Wang in 1989, in the context of cellu lar robotic systems. Swarm intelligence is "The emergent collective intelligence of groups of simple agents" (Bonabeau et al., 1999). It gives rise to complex and often intelligent behavior through simple, unsupervised interactions between a total numbers of autonomous swarm members. Usually there is no centralized control structure dictating how the individual agents should behave, but local interactions between such agents often lead to the emergence of a global behavior.

Swarm is considered as biological insects like ants, bees, wasps, fish etc. The quick coordinated flight of a group of birds with very little visual communication and the concerted effort of an ant colony in gathering food, building nests, etc are some of the vivid examples of emergence in natural world. SI has found immense applicability in fields like Robotics, Artificial Intelligence, process optimization, telecommun ications, routing, software testing, networking etc. The following two subsections will describe about ant colonies and bee colonies and their mechanis ms.

\subsection{Ant Colony System}

The phenomenon of emergence found in natural systems show how simple behavioural patterns from participants give rise to complex self-regulatory behaviour of the entire system[2]. Ant Colony Optimization (ACO) [3] is a branch of a newly developed form of Artificial Intelligence called Swarm Intelligence. In groups of insects, which live in colonies, such as ants and bees, an individual can only do simple tasks on its own, which the colony's cooperative work is main reason determining the intelligence behaviour it shows. Figure 1 shows a scenario in which the best route between two choices is chosen by the ants. 
a)

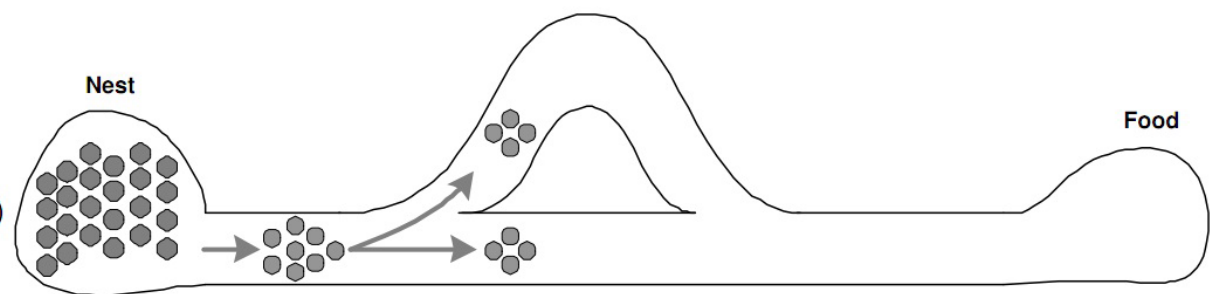

b)
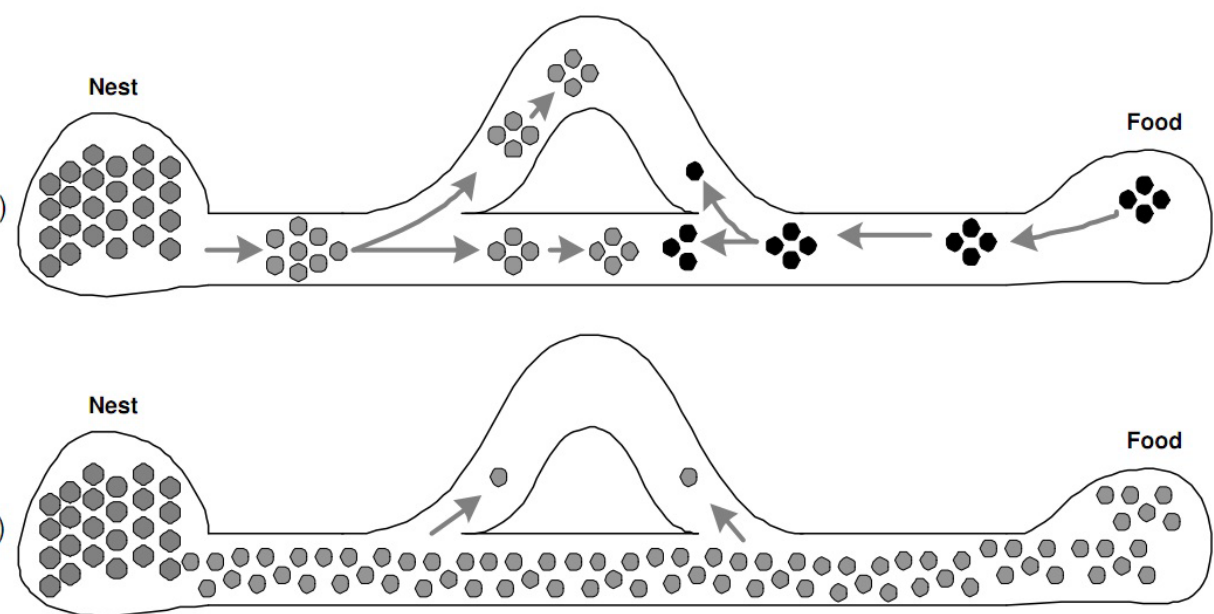

Fig. 1: All ants take the shortest path after an initial searching time

Ant colony optimization (ACO) [4] is an optimization technique inspired by the exploratory behaviour of ants while finding food. The ants in the colony form a collective behaviour. A colony of ants has a wide range of duties like collecting food, building/guarding the nest, removing the dead ants, etc and has simple one-to-one communication. The individual messages passed between ants are very insignificant, but the collective messages help in coordinated work control of ants without the presence of a centralized control system. Ants use scent called pheromone for communication among them. This is a chemical substance deposited on the ground, by ants when they roam around for search of food. Ants have ability to smell this pheromone. They can produce few different types of pheromones - usually one each to signify different work categories like collecting food trails, signifying emergency, moving dead ants, etc. The source of ACO is the pheromone trail laying and following behaviour of ants which use pheromone as a communication medium.

While searching the environment for food, the ants deposit pheromones on the ground. Other ants are attracted by pheromones and tend to follow trails of previous ants. This mechanism enables the ants to find shortest paths between the nest and a food source. When ants fan out to find food, it may happen that a lucky ant finds a short path to a new food source. It then takes some food with it and makes its way back to the nest. Since it is attracted by its own pheromone trail, it is likely that the ant follows its own path back to the nest, thereby leaving a second pheromone trail. If other ants happened to take a longer path to the food source, they arrive after the first ant and, when trying to make their way back to the nest, there is a good chance for them to be attracted by the short path, where already two pheromone trails have been laid. This reinforces the short path even more and makes it more attractive. Concerning the longer path, pheromones tend to evaporate after some time, so in the long run the long paths will be forgotten and almost all ants will take the short path. The characteristics of ants are similar to the characteristics of MANETs. This helps us to apply the food searching characteristics of ants for routing packets in MANETs.

The basic principle of an ant routing algorithm is mainly the depositing of pheromone on the path followed by the ant. They follow simple rule of following the path which has higher concentration of pheromone. The pheromone concentrations on a path allow the other ants to find their way to the food source. Thereby more ants follow the same path and more and more pheromone is deposited on the path which is the shortest route to the food source. It was found that the pheromone-trail-following behavior gives rise to the emergence of the shortest path which is followed by other ants of the colony. When a previously short route get blocked/ lengthened due to an obstacle en route, the alternate short route get strengthened with higher pheromone content due to shorter end-to-end travel time and more ants move to this route. Hence the path can also dynamically adapt to fast changes in the environment.

On the basis of Figure 2 (a), (b) and (c) in comparison with routing of packets in MANETs, we can define the Ant routing basic principle as: 
1. Each network node sends a number of discovery packets that is, called forward ants (F-ANT) are sent towards the selected destination nodes of the network as shown in Figure 2(a).

2. The routing tables maintained at each node are replaced with stochastic tables, which select next hops according to weighted probabilities available.

3. Accordingly, the ants deposit pheromone on the crossed links, that is, in the nodes routing tables are changed for selection of the next node in the network.

4. When forward ant (F-ANT) reaches the destination node, it generates a backward ant (B-ANT) and then dies. Similarly in MANETs routing, the new packet created and sent back to the source will propagate through the same path selected by the forward ant (F-ANT). This is shown in Figure 2(c).

5. Now backward ant (B-ANT) deposits pheromone on the crossed links. It means that it updates the routing table of the nodes along the path followed by forward ant (F-ANT).

6. After arrival to the source node, the backward ant (B-ANT) dies.

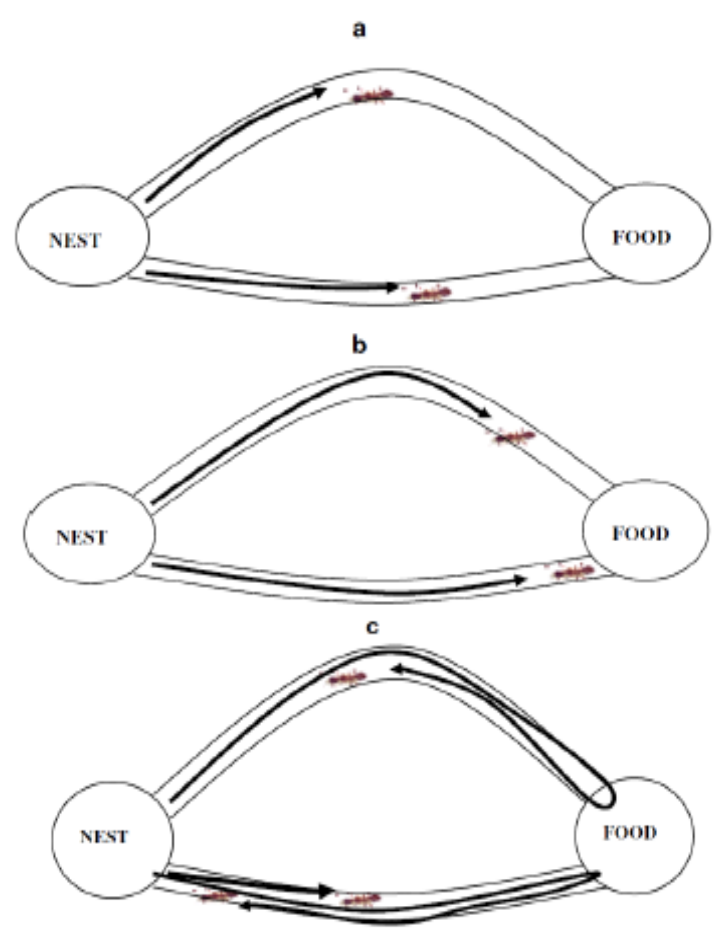

Fig. 2: Basic ant routing principle

\subsection{Bee Colony System}

Honey bee colonies have attracted a strong interest as a potential source of inspiration for the design of optimization strategies for dynamic, time-vary ing, and multi-objective problems. Bee colonies show structural characteristics similar to those of ant colonies. Bees utilize a sophisticated communication protocol that enables them to communicate directly through bee-tobee signals and when required, similar to ants, use stigmergic feedback cues for bee-to-group or group-to- bee communication. In these two classes of insects, communication and cooperation is realized according to radically different modalities due to the different nature of these insects (ants mainly walk, while bees mainly fly). In the case of ants communication is achieved via a pheromone trail that is laid on the ground while walking, in the case of bees it is a form of visual communication that plays an equivalent role.

Bee Colony Optimization (BCO) [5] is the SI system where the low level agent is the bee. $\mathrm{BCO}$ is the name given to the collective food forag ing behavior of honey bee. The bee system is a standard example of organized team work, well coordinated interaction, coordination, labor division, simultaneous task performance, specialized individuals, and well-knit communication. In a bee colony there are different types of bees like a queen bee, many male drone bees and thousands of worker bees. The Queen's responsibility is of laying eggs so that new colonies can be formed. The Drones are males of the hive and are responsible to mate with the Queen. This is their sole role in the hive. The worker bees are the females of the hive. They are the main build ing blocks of the hive. They build the honey bee comb, clean it, maintain it, guard it and feed the queen and drones. In addition to these, the main job of a worker bee is to search and collect rich food. There are two types of worker bees namely scout bees and forager bees. The Scout bees fly around and search for food sources available randomly. They return back to the hive after they exhaust their energy and distance limits. Upon returning to the hive they share their exploration experience and a lot of important information with the forager bees. The scouts tell the foragers about the location of rich food sources which comprises of the direction of the food source from the hive with respect to sun and distance from hive. Th is is done using a dance called "waggle dance" which is in the figure of digit " 8 ". It also indicates the quality of food. The forager bee closely observes the scout bee to learn the directions and information given by scout and then goes to collect food.

\section{Ant Inspired Routing Protocols}

The main characteristic of an ACO routing algorith m [6] consists in the continual acquisition of routing information through path sampling and discovery using small control packets called artificial ants. This is to learn statistical estimates of the quality of each local routing choice. The ants are generated concurrently and independently by the nodes, with the task to try out a path to an assigned destination. An ant going from source $s$ to destination $d$ collects information about the quality of the path, and, either on the way to $d$ or while return its way back from $\mathrm{d}$ to $\mathrm{s}$, it uses this information to update the routing tables at intermediate nodes, reinforcing the good paths. The routing table at node $\mathrm{i}$ is derived from the pheromone table. So me of the ACO based routing protocols found in the literature are Ant 
Based Colony (ABC) in 1997 by Ruud Schoonderwood, ANTNET in 1997 by Gianni Di Caro, ANT-AODV in 2002 by Shrivanajay Marwala, Chen kong Tham, Dipti Shrinavasan, TERMITE in 2003 Martin Roth and Stephen Wicker, ANTHOCNET in 2004 by Gianni Di Caro, Frederick Ducatelle LM Gambardella and POSANT in 2008 by Shabab Kamali and Jaroslav Optarny are few of them. In the next three sub sections, we have studied three latest protocols found in the literature.

\subsection{AntNet-RSLR:}

Ahmed A. A. Radwan [7] proposed a novel routing scheme for MANETs, which adapts the AntNet protocol with the blocking-expanding Ring Search and Local Retransmission technique (AntNet-RSLR). According to the AntNet-RSLR, the packets used in the network can be divided into two classes - data packets and control packets. Data packets represent the information that the end-users exchange with each other. In ant-routing, data packets use the information stored at routing tables for travelling from the source to the destination node. AntNet-RSLR contains a special routing table, in which each destination is associated to all interfaces and each interface has a certain probability. Forward ant (FANT) and a backward ant (BANT) control packets are used to update the routing tables and distribute information about the traffic load in the network. In addition to the above, the neighbour control packets are used to maintain a list of available nodes to which packets can be forwarded.

In this algorithm, in the route discovery phase new routes are created by FANT and BANT. A FANT is a small packet with a unique sequence number establishes the pheromone track to the source node. It gathers information about the state of network. A BANT establishes the pheromone track to the destination node. In route maintenance phase, the routes need to be monitored and strengthened during the communication. Once the FANT and BANT have established the pheromone tracks for the source and destination nodes, subsequent data packets are used to maintain the path. AntNet-RSLR recognizes a route failure through a missing acknowledgement. If a node gets a route error (RERR) message for a certain link, it deactivates this link by setting the pheromone value to 0 . Then the node searches for an alternative lin $\mathrm{k}$ in its routing table.

According to this protocol, a group of mobile agents build paths between pair of nodes, exploring the network concurrently and exchanging obtained information to update the routing tables that decreases both of the routing message overhead and the average end to end delay less than the well known AntNet, AODV and DSR routing protocols. This led to increase the throughput more than AntNet, AODV and DSR routing protocols.

\subsection{SAMP-DSR:}

Switched ACO based MP-DSR (SAMP-DSR) [8] is based on Multi-Path Dynamic Source Routing introduced in [9] and Enhanced Multi-path Dynamic Source Routing (EMP-DSR) algorithm proposed in [10], which uses ants pro-actively to feed its re-active path discovery process with global data. The approach presented in [8] is a QoS-aware routing protocol, considering end-to-end reliability parameter as a constraint while trying to minimize other parameters such as delay.

There are two important components in SAMP-DSR. Proactive ACO-based component is the first component, which run all time in the background and the second is the reactive path discovery component which is triggered upon a route request. Apart from the above, SAMP-DSR has two working modes, ACO mode and local mode. ACO-based proactive component plays a dominant role in providing global information and path selection preferences in ACO mode. In local mode the decisions are based on local information which each node gathers solely from its own neighbours. The goal of local mode is to stop or minimize the overhead of ACO based proactive component when it cannot perform as expected in highly dynamic network environments. The SAMPDSR behaves similar to EMP-DSR on lower mobility modes and to MP-DSR as network becomes more mobile.

\subsection{ODASARA:}

A novel On Demand Ant based Security Alert Routing Algorithm (ODASARA) for mobile ad hoc a network in grid environment is proposed in [11]. This protocol combines the Ad Hoc On-Demand Distance Vector (AODV) routing protocol with Ant Colony Optimization mechanism using ant like mobile agents.

In ODASARA, a security metric is embedded into the RREQ packet, and forwarding behaviour of the protocol is changed with respect to RREQs. Intermediate nodes receive the RREQ packet with security metric. The Security Alert Routing (SAR) ensures that this node can only process the packet or forward it if the node itself can provide the required security or trust level, otherwise the RREQ is dropped. If an end-to-end path with the required security attributes can be found, a suitably modified RREP is sent from an intermediate node or the eventual destination. The route discovered by ODASARA between two communicating entities may not be the shortest route in terms of hop-count, but with a quantifiable guarantee of security. If one or more routes that satisfy the required security attributes exist, SAR will find the shortest route. If all the nodes on the shortest path between two nodes can satisfy the security requirements, this protocol will find optimal routes. However, if the ad hoc network does not have a 
path with nodes that meet RREQ's security requirements, SAR may fail to find a route.

There are different techniques to measure or specify the quality of security of a route discovered by SAR protocol. The first technique is the explicit representation of trust levels using a simple hierarchy. ODASARA provides applications the ability to incorporate explicit trust levels into the route discovery process. Another alternative is to use what we call the QoP (Quality of Protection) bit vector.

Threats to information in transit include corruption of information, disclosure of sensitive information, theft of legitimate service from other protocol entities, or denial of network service to protocol entities. In SAR, the messages are protected by the key management infrastructure. ODASARA provides digital signatures and encryption techniques that can be incorporated on a need-to-use basis to prevent modification.

\subsection{Hybrid Ant Colony Optimization Routing Algorithm for Mobile Ad Hoc Network (HOPNET):}

HOPNET [12] is a hybrid routing algorithm for MANETs based on ACO and zone routing framework of broadcasting. This algorithm is based on ants hopping from one zone to the next, consists of the local proactive route discovery within a node's neighborhood and reactive communication between the neighborhoods. The algorithm has features extracted from ZRP and DSR protocols. The network is divided into zones which are the node's local neighborhood. The size of the zone is determined by the radius length measured in hops.

Therefore, a routing zone consists of the nodes and all other nodes within the specified radius length. The nodes are categorized as interior nodes and boundary nodes. Boundary nodes are at a distance from the central node. All other nodes less than the radius are interior nodes. The following figure 3 is an example for HOPNET.

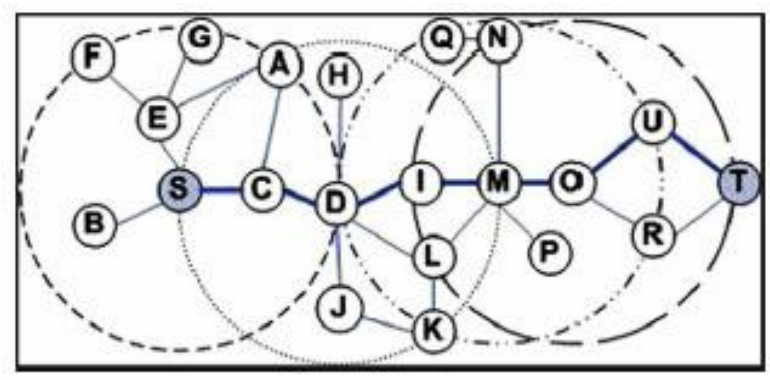

Fig. 3: HOPNET example

Suppose, if the radius of the zone is 2 then for node S, nodes A, D, F, G are boundary nodes, and nodes B, E, $\mathrm{C}$ are interior nodes. According to this algorithm each node maintains two routing tables: Intrazone Routing Table (IntraRT) and Interzone Routing Table
(InterRT). The IntraRT is proactively maintained and a node can obtain a path to any node within its zone quickly. After a forward ant reaches destination, a corresponding backward ant is sent back along the path discovered. The InterRT stores the path to a node beyond its zone. This source routing table is setup on demand as routes outside a zone is required. The peripheral nodes of the zone are used to find routes between zones.

To discover the route, the network can be represented as a graph $\mathrm{G}(\mathrm{V}, \mathrm{E})$ where $\mathrm{V}$ is the number of vertices and $\mathrm{E}$ is the number of edges. The ACO algorith $m$ is used to find the shortest or best path from source to the destination. Each link e $=(v i, v j)$ is associated with two variables: $\varphi(v i, v j)$ represents the pheromone value on each link and $\mathrm{w}(\mathrm{vi}, \mathrm{vj})$ represents the time. The pheromone value gets updated by the ants as they traverse the links. The ants change the concentration of the pheromone value on their journey to the destination and on their way back to the source.

\section{Bee Inspired Routing Protocols}

Bee Colony Optimization (BCO) can be applied to software testing, puzzle solving, numerical comple xity problems, routing problems, networking problems, assignment problems, optimization problems, accident diagnosis etc. BCO has immense capabilities to solve problems with minimum scope to solution. In the area of routing protocols for ad hoc networks, researches have proposed few protocols based on BCO, which include Artificial Bee Colony (ABC), BeeAdHoc protocol etc.

\subsection{BeeAdHoc:}

BeeAdHoc is a nature inspired routing protocol for MANETs based on the foraging principles of honey bees [13]. It mainly utilizes two types of agents scouts to discover routes and foragers to transport data. Figure 4 will give an overview of the BeeAdHoc architecture. In this each node maintains a hive with an Entrance, Packing Floor and a Dance Floor.

Entrance provides an interface to the Media Access Control (MAC) layer of the network stack and handles all incoming/outgoing packets. A scout received at the entrance is broadcasted further if its time to live (TTL) timer has not expired or if it has not arrived at the destination. The information about the id of the scout and its source node is stored in a table. If another replica of an already received scout arrives at an entrance of a hive then the new replica is killed. If a forager with a same destination as that of the scout already exists in the dance floor then the route to the destination is given to the scout by appending the route in the forager to its current route. If the current node is the destination of a forager then it is forwarded to the packing floor else it is directly forwarded to the MAC interface of the next hop node. 


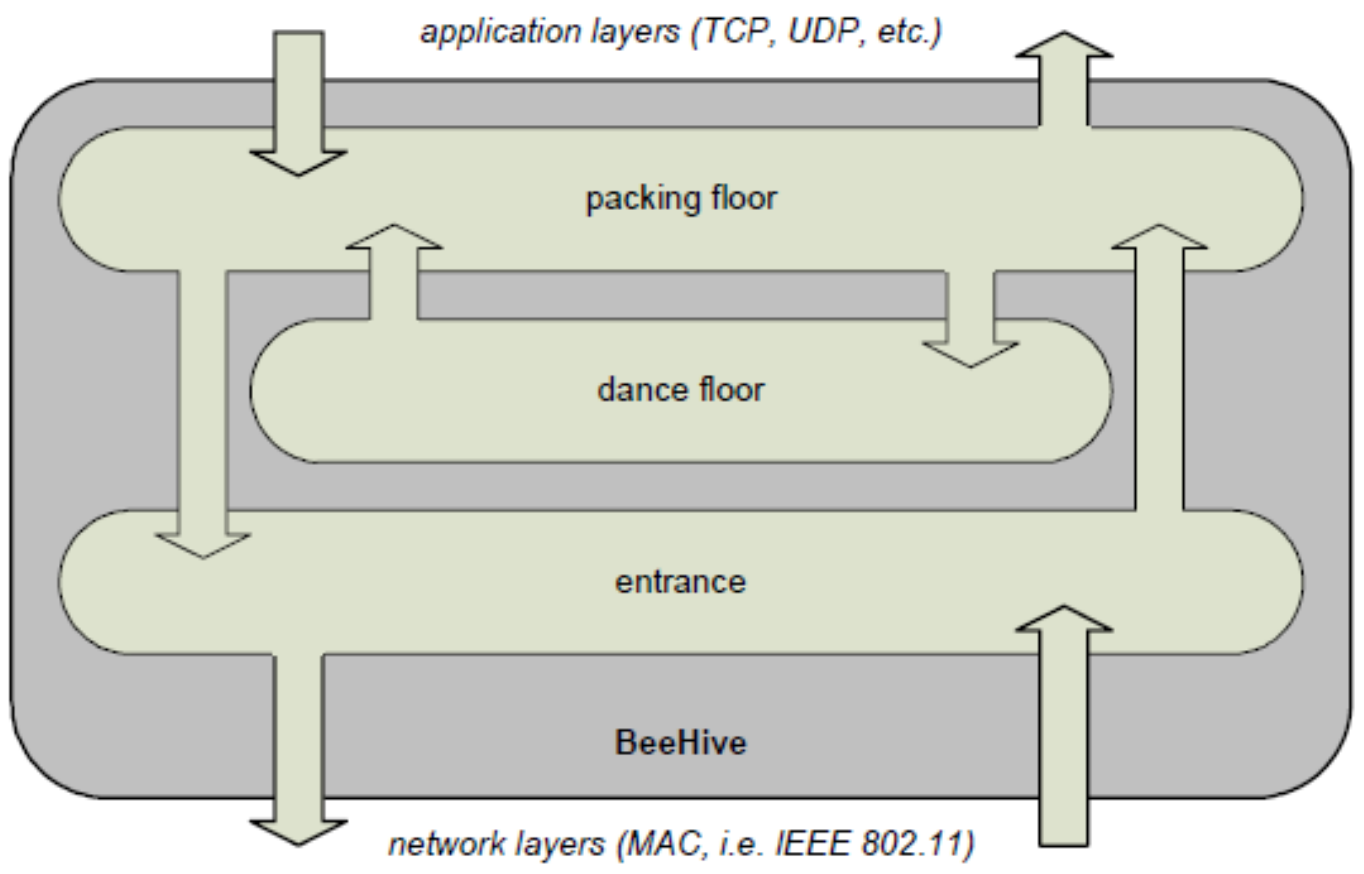

Fig 4: Overview of the BeeAdHoc architecture

Packing floor is an interface to the transport layer and receives data from it. Once a data packet arrives from the transport layer, a packer is created in this floor which stores the data packet. After that the packer tries to locate a suitable forager for the data packet from dance floor. If it finds the forager then it handovers the data packet to it and dies.

The dance floor is the heart of the hive because it takes important routing decisions. Once a forager returns after its journey it recruits new foragers by dancing according to the quality of path that it traversed. A lifetime forager evaluates the quality of its route based on the average remaining battery capacity of the nodes on its route. A lifetime forager might allow itself to be cloned many times in two scenarios: one, the nodes on the route have enough remaining battery capacity (good route), two, if large number of packers are waiting for it even though its route might be having nodes with little battery capacity. On the other hand, if none of the packers are waiting then a forager with a very good route might not dance because its colleagues are doing a nice job in transporting the data packets. This concept is directly borrowed from the behavior of scout/forager bees in Nature, and it helps in regulating the number of foragers for each route.

\section{Conclusion}

Lot of research has been done and shown that nature inspired routing protocols can remove at least one or several problems in the area such as battery life, scalability, maintainability, survivability, adaptability and so on. In this paper we have briefly introduced the nature inspired routing algorithms for MANETs derived from the ant colonies and bee colonies. The agents in Ant Colony inspired routing algorithms communicate indirectly through the stigmergy and the agents provide positive feedback to a solution by laying pheromone on the links. Moreover, they have negative feedback through evaporation and aging mechanis ms, which avoids stagnation. From our study it was observed that the important advantage of BeeAdHoc is the distribution of the traffic to different routes proportional to their quality and capacity. This has the absence of many control packets compared to other algorithms and are limited to the scouts. The most important disadvantage of BeeAdHoc is the use of source routing. This also appears quite unnatural compared to real bees, because they use vector guidance. The disadvantage in computer networks comes from the control overload per packet and the limitation of the maximal route length. It uses the higher memory for storing every forager. It can be concluded that Swarm Intelligence based approach offers to be a powerful means to solve routing problems in Mobile Ad hoc Networks. Further, it is required to consider real scenario/ constraints/ environmental conditions and need to tune and simulate to get an efficient and effective routing protocols for Mobile Ad hoc Networks.

\section{References}

[1] E. Bonabeau, M. Dorigo, and G. Theraulaz, Swarm Intelligence - From Natural to Artificial Systems. New York: Oxford University Press, 1999. 
[2] G. Serugendo, N. Foukia, S. Hassas, A. Karageorgos, S. K. Mostefaoui, O. F. Rana, M. Ulieru, P. Valckenaers, C. Van Aart, "Selforganisation: Paradig ms and applications", Engineering Self-Organizing Systems, Vol. 2977, 2004

[3] Dorigo, M., \& Caro, G.D 1999. Ant Algorithms for Discrete Optimization. Artificial Life.

[4] Dorigo M. and G. Di Caro. Ant colony optimization: a new meta-heuristic. In Proceedings of the Congress on Evolutionary Computation, 1999.

[5] Dr. Arvinder Kaur, Shivangi Goyal. A Survey on the Applications of Bee Colony Optimization Techniques in International Journal on Computer Science \& Engineering 2011.

[6] G.A. Di Caro, F. Ducatelle, and L.M. Gambardella. Theory and practice of Ant Colony Optimization for routing in dynamic telecommunications networks. In N. Sala and F. Orsucci, editors, Reflecting interfaces: the complex co evolution of information technology ecosystems. Idea Group, Hershey, PA, USA, 2008.

[7] Ahmed. A. A. Radwan, Tarek. M. Mahmoud, Essam. H. Hussein AntNet-RSLR: A Proposed Ant Routing Protocol for MANETs 2011.

[8] Ehsan Khosrowshahi-As1, Majid Noorhosseini And Atieh Saberi Pirouz A Dynamic Ant Colony Based Routing Algorithm for Mobile Ad-hoc Networks Journal Of Information Science And Engineering, 1581-1596 2011.

[9] S. S. Dhillon, X. Arbona, and P. V. Mieghem, "Ant routing in mobile ad hoc networks," in Proceedings of International Conference on Networking and Services, 2007, pp. 67.

[10] E. Khosrowshahi-Asl, M. Damanafshan, M. Abbaspour, M. Noorhosseini, and K.Shekoufandeh, "EMP-DSR: An enhanced multi-path dynamic source routing algorithm for MANETs based on ant colony optimization," in Proceedings of the 3rd Asia International Conference on Modeling and Simulation, 2009, pp. 692-697.

[11] R.Rameshkumar, Dr. A.Damodaram, ODASARA: A Novel on Demand Ant Based Security Alert Routing Algorithm for MANET in Grid Environment, IJCSNS International Journal of Computer Science and Network Security, April 2010.

[12] Jianping Wang, Eseosa Osagie, Parimala Thulasiraman, Ruppa K. Thulasiram HOPNET: A hybrid ant colony optimization routing algorithm for mobile ad hoc network Elsevier 2008.

[13] H.F.Wedde, M. Farooq, T. Pannenbaecker, B. Vogel, C. Mueller, J. Meth, and R. Jeruschkat.
Beeadhoc: an energy efficient routing algorithm for mobile ad hoc networks inspired by bee behavior. In GECCO, pages 153-160, 2005.

[14] Charles E. Perkins, Ad Hoc Networking, Addison-Wesley, 2001.

[15] C.K. Toh, Ad Hoc Mobile Wireless Networks: Protocols and Systems, Prentice Hall, 2001.

[16] R. Bhaskar, J. Herranz, and F. Laguillaumie, "Efficient authentication for reactive routing protocols," in AINA '06: Proceedings of the 20th International Conference on Advanced Information Networking and Applications Volume 2 (AINA'06). Washington, DC, USA: IEEE Computer Society, 2006, pp. 57-61.

[17] C.Perkins and P.Bhagwat, "Highly dynamic destination-sequenced distance-vector routing (dsdv) for mobile computer," ACM Sigcomm'94, August 1994.

[18] Perkins C.E. and Royer E. (1999) 'Ad-hoc ondemand distance vector routing', Proceedings of the 2nd IEEE Workshop on Mobile Computing Systems and Applications, New Orleans, USA, pp. 90-100.

[19] Haas Z.J., Pearlman M.R. and Samar P.(2002) 'The zone routing protocol (ZRP) for ad hoc networks', IETF Internet Draft, draft-ietfmanetzone-zrp-04.txt.

[20] Y. B. Ko and N. H. Vaidya, "Location-Aided Routing (LAR) in Mobile Ad Hoc Networks," Proc. ACM/IEEE MOBICOM '98, Oct. 1998.

[21] Baras J. and Mehta. H. (2003) 'A Probabilistic Emergent Routing Algorithm for Mobile Ad hoc Networks', Proceeding of workshop on Modeling and Optimization in Mobile, Ad Hoc and Wireless Networks, pp 20-24.

[22] M. Genes, U.Sorges, and I.Bouazizi. ARA - the ant-colony based routing algorithm for manets. In Proceedings of ICPP Workshop on Ad Hoc Networks, 2002.

[23] R. Schoonderwoerd, O.E. Holland, J.L. Bruten, and L.J.M. Rothkrantz. Ant-based load balancing in telecommunications networks. Adaptive Behavior, 5(2):169-207, 1996.

[24] G.Di Caro and M.Dorigo. AntNet: Distributed stigmergetic control for communication networks. Journal of Artificial Intelligence, 9:317-365, December 1998.

[25] Gianni Di Caro, Frederick Ducatelle, and Luca Maria Gambardella. AntHocNet: an ant-based hybrid routing algorithm for mobile ad hoc networks. In Proceedings of Parallel Problem Solving from Nature (PPSN) VIII, LNCS 3242. Springer-Verlag, 2004. 
[26] Martin Roth and Stephen Wicker. Termite: Emergent ad-hoc networking. In Proceedings of the Second Mediterranean Workshop on Ad-Hoc Networks, 2003.

[27] H.F. Wedde, M. Farooq, and Y. Zhang. Beehive: An efficient fault-tolerant routing algorithm inspired by honey bee behavior. In Proceedings of ANTS Workshop, LNCS 3172, pages 83-94. Springer Verlag, Sept 2004.

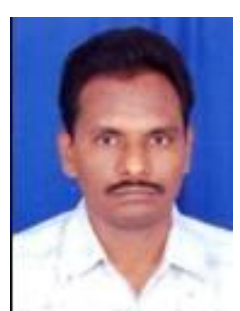

CH.V. Raghavendran has received MCA and M.Tech (CSE) degrees from Nagarjuna University in 1994 and 2010 respectively. He received his M.Phil in Computer Science in 2008 from Alagappa University. He is a research scholar in Compute Science Department of Adikavi Nannaya University, Rajahmundry, AP. He has published over 10 papers in various National and International Conferences. He is working as a Director of P.G. Dept. of Computer Sciences, Ideal College of Arts \& Sciences, Kakinada, AP. His areas of interest are Mobile Ad hoc Networks, Swarm Intelligence and Data Mining.



Ganti Naga Satish is working as Associate Professor in P.G. Department of Computer Sciences, Ideal College of Arts \& Sciences, Kakinada, Andhra Pradesh, India. His qualifications are M.Sc, M.Phil, M.Tech. He is pursing Ph.D at Adikavi Nannaya University. He has presented and published papers in National and International Conferences. His areas of interest include Computer Networks.

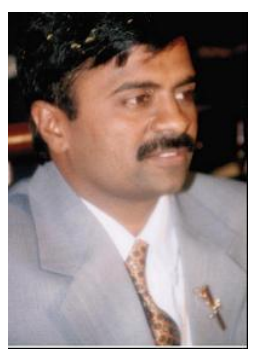

Dr. P. Suresh Varma received the Master's degree M.Tech in Computer Science \& Technology from Andhra University. He received $\mathrm{Ph} . \mathrm{D}$. degree in Computer Science \& Engineering from Acharya Nagarjuna University. He is currently working as Professor in Department of Computer Science in Adikavi Nannaya University, Rajahmundry, A.P., India. He published several papers in National and International Journals. He is active member of various professional bodies. His current research is focused on Computer Networks, Cloud Computing and Data Mining.
How to cite this paper: CH. V. Raghavendran, G. Naga Satish, P. Suresh Varma,"Intelligent Routing Techniques for Mobile Ad hoc Networks using Swarm Intelligence", International Journal of Intelligent Systems and Applications(IJISA), vol.5, no.1, pp.81-89, 2013.DOI: 10.5815/ijisa.2013.01.08 